\title{
A Model for Coronary Heart Disease and Stroke With Applications to Critical Illness INSURANCE UNDERWRITING II: APPLICATIONS
}

\author{
Angus S. Macdonald, ${ }^{*}$ Howard R. Waters, ${ }^{\dagger}$ and Chessman T. Wekwete ${ }^{\ddagger}$
}

\begin{abstract}
In Part I we constructed a model for the development of coronary heart disease (CHD) or stroke that either incorporates, or includes pathways through, the major risk factors of interest when underwriting for critical illness (Cl) insurance. In Part II we extend this model to include other critical illnesses, for example, cancers and kidney failure, and describe some applications of the model. In particular, we discuss $\mathrm{Cl}$ premium ratings for applicants with combinations of some or all of high body mass index, smoking, high blood pressure, high cholesterol, and diabetes. We also consider the possible effect on $\mathrm{Cl}$ premium ratings of genetic conditions that increase the likelihood of high blood pressure, high cholesterol, diabetes, CHD event, or stroke.
\end{abstract}

\section{INTRODUCTION}

In Part I we formulated a model for the development of coronary heart disease (CHD) and stroke, incorporating risk factors usually taken into account by insurance underwriters, namely, age, sex, smoking status, body mass index (BMI), hypertension, hypercholesterolemia, and diabetes. The model was parameterized to a large extent using data from the Framingham Heart Study. In this part we extend the model so that it can be used as a model for critical illness (CI) insurance.

In Section 2 we describe our extended model, and in Sections 3 and 4 we describe the parameterization of the additional transition intensities required by our model. In Section 5 we discuss premiums and premium ratings for CI insurance calculated using our model. In Section 6 we show some sample calculations designed to assess the likely impact on CI premiums of genetic information relating to CHD or stroke or any of our three risk factors.

\section{The Critical Illness Model}

Our model for CI insurance is shown as Figure 1. This is similar to, but an extension of, the model shown as Figure 3 in Part I. Like the earlier model, Figure 1 is a continuous-time, time-inhomogeneous Markov process, which represents an individual's progress toward a set of absorbing states, with time being equivalent to the individual's age. A difference from the earlier model is that this new model has four, rather than three, absorbing states-a CHD event, stroke, diagnosis of any other disease that would qualify for benefit to be paid from a CI policy, and death-the additional state being the other critical illnesses. Progress toward the absorbing states is through the transient states that represent combinations of categories of hypertension, hypercholesterolemia, and diabetes. The model developed

\footnotetext{
* Angus S. Macdonald, FFA, PhD, is a Professor in the Department of Actuarial Mathematics and Statistics, School of Mathematical and Computer Sciences, Heriot-Watt University, Edinburgh, EH14 4AS, Scotland, U.K., e-mail: A.S.Macdonald@ma.hw.ac.uk.

${ }^{\dagger}$ Howard R. Waters, FIA, FFA, DPhil, is a Professor in the Department of Actuarial Mathematics and Statistics, School of Mathematical and Computer Sciences, Heriot-Watt University, Edinburgh, EH14 4AS, Scotland, U.K., e-mail: H.R.Waters@ma.hw.ac.uk.

¥ Chessman T. Wekwete, PhD, is a Research Actuary (Life and Health) at Swiss Re—South Africa, P.O. Box 72209, Parkview 2122, South Africa, e-mail: Chessman_Wekwete@swissre.com.
} 
Figure 1

CHD/Stroke Model for Critical Illness Insurance

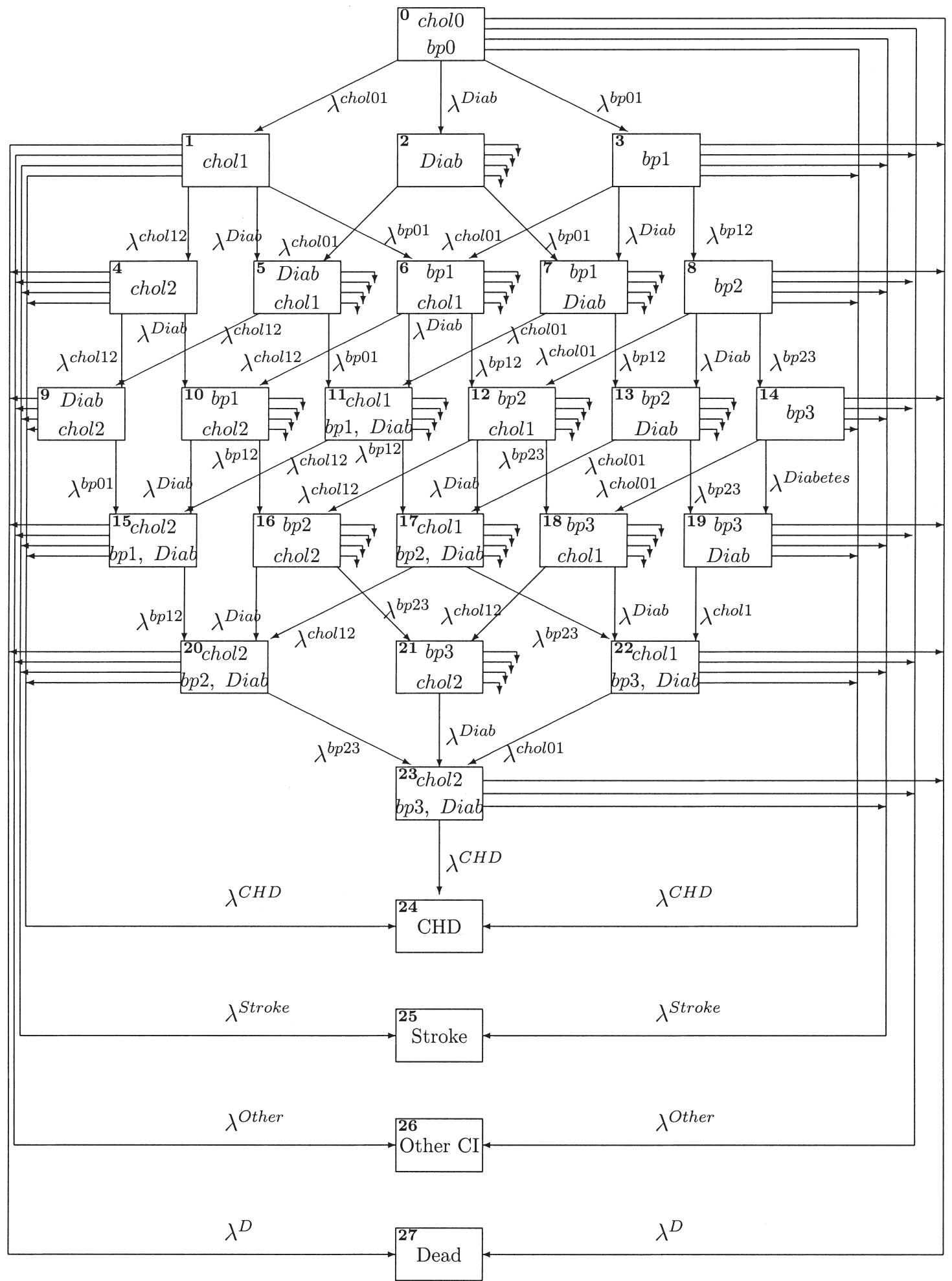


in Part I had $24(=4 \times 3 \times 2)$ transient states. We will see following Section 3 that the model for CI in Figure 1 has 36 transient states; the difference being that boxes labeled "Diab" in Figure 1 actually represent two separate boxes, one for each of Type 1 and Type 2 diabetes.

As for the model in Part 1, there are 12 separate parameterizations of the extended model, one for each of the subpopulations determined by combinations of sex (2), smoking status (2), and BMI (3). The intensities for the transitions between the transient states and from these states to the absorbing states "CHD event" and "stroke" for the new model are exactly the same as for the earlier model. See Sections 8,9 , and 10 of Part I. The parameterization of the incidence of other critical illnesses and of the force of mortality is described in Sections 3 and 4.

\section{The Intensities for the Other Critical Illnesses}

In this section we discuss the parameterization of the intensities of being diagnosed with a critical illness other than CHD or stroke. These illnesses typically include cancers, kidney failure, and total and permanent disability. We assume that all these intensities depend on age and sex but, with two exceptions, not on the presence or absence of any of the other risk factors. The two exceptions are lung cancer, where smoking status is relevant, and kidney failure, where some diabetics have an increased risk.

\subsection{Cancers Other than Lung Cancer}

Data from the Office of National Statistics (ONS 1998) give for individual ages and separately for each sex, the number of cases of cancer, excluding lung cancer and malignant melanoma, in 1990-92. The corresponding exposures can be found in OPCS (1991, 1993a, 1993b). We smoothed the crude incidence rates for these cancers for females and males using weighted (by exposure) least squares, giving the following intensities:

$$
\begin{aligned}
& \lambda_{x}^{\text {othercanfemales }}= \begin{cases}\exp \left(\alpha_{0}+\alpha_{1} \times x+\alpha_{2} \times x^{2}\right) & : x \leq 52 \\
\exp \left(\beta_{0}+\beta_{1} \times x+\beta_{2} \times x^{2}\right) & : x>52\end{cases} \\
& \lambda_{x}^{\text {othercan_males }}= \begin{cases}\exp \left(\alpha_{0}+\alpha_{1} \times x\right) & : x \leq 55 \\
\exp \left(\beta_{0}+\beta_{1} \times x+\beta_{2} \times x^{2}\right) & : x>60\end{cases}
\end{aligned}
$$

with linear blending between ages 55 and 60 for males. The coefficients for these functions are given in Table 1.

\subsection{Lung Cancer}

The data from the ONS and the OPCS used in the previous section also give the number of cases of lung cancer for individual ages and for each sex, but not differentiated by smoking status. Using these data we fitted, using weighted least squares, the following functions to the incidence rates of lung cancer for males and for females:

Table 1

Coefficients for Fitting "Other Cancers" Incidence

\begin{tabular}{|c|c|c|}
\hline Coefficient & Females & Males \\
\hline$\alpha_{0}$ & -11.78 & -11.02 \\
$\alpha_{1}$ & $1.773 \times 10^{-1}$ & $9.621 \times 10^{-2}$ \\
$\alpha_{2}$ & $-1.052 \times 10^{-3}$ & -16.37 \\
$\beta_{0}$ & -8.510 & $2.725 \times 10^{-1}$ \\
$\beta_{1}$ & $7.262 \times 10^{-2}$ & $-1.443 \times 10^{-3}$ \\
\hline$\beta_{2}$ & $-2.560 \times 10^{-4}$ & \\
\hline
\end{tabular}




$$
\begin{aligned}
& \lambda_{x}^{\text {lung_males }}= \begin{cases}\exp \left(\alpha_{0}+\alpha_{1} \ln (x)+\alpha_{2} \ln (x)^{2}\right) & : x \leq 60 \\
\exp \left(\beta_{0}+\beta_{1} \ln (x)+\beta_{2} \ln (x)^{2}\right) & : x>60\end{cases} \\
& \lambda_{x}^{\text {lung_females }}= \begin{cases}\exp \left(\alpha_{0}+\alpha_{1} \ln (x)+\alpha_{2} \ln (x)^{2}\right) & : x \leq 59 \\
\exp \left(\beta_{0}-\exp \left(\beta_{1}+\beta_{2} \ln (x)\right)\right) & : x>65\end{cases}
\end{aligned}
$$

with linear blending for females between ages 59 and 65. The coefficients are given in Table 2.

Doll (1971, Table 6) gives data (for males) from which we can estimate the relative risk of lung cancer for smokers and nonsmokers. Doll's data show that the relative risk is about 10:1 at age 47 and about 20:1 at age 62. We model this relative risk by the function $\operatorname{rr}(x)$, which we assume applies to females as well as males, where

$$
r r(x)= \begin{cases}1, & \text { for } x \leq 33.6 \\ -21.5+0.67 x, & \text { for } x>33.6\end{cases}
$$

We assume that $34 \%$ of males and $31 \%$ of females are smokers, and that these percentages apply at all adult ages. These percentages are based on data for adults in England in 1986 available from the Department of Health (1998, Table 5). (Using data from earlier than the lung cancer incidence data [1992] is appropriate since there is a time lag between smoking and the development of lung cancer.) Using these percentages, we obtain the following the incidence rates of lung cancer, as functions of age, split by sex and smoking status:

$$
\begin{gathered}
\lambda_{x}^{\text {lung_males_non-sm }}=\lambda_{x}^{\text {lung_males }} \times(0.34 \times r r(x)+0.66)^{-1}, \\
\lambda_{x}^{\text {lung_males_sm }}=\lambda_{x}^{\text {lung_males }} \times \operatorname{rr}(x) \times(0.34 \times r r(x)+0.66)^{-1}, \\
\lambda_{x}^{\text {lung_females_non-sm }}=\lambda_{x}^{\text {lung_females }} \times(0.31 \times r r(x)+0.69)^{-1}, \\
\lambda_{x}^{\text {lung_females_sm }}=\lambda_{x}^{\text {lung_females }} \times \operatorname{rr}(x) \times(0.31 \times r r(x)+0.69)^{-1} .
\end{gathered}
$$

Figure 2 shows a graph of the incidence rates for males, smokers and nonsmokers, as well as the observed population rates. The population rates are for smokers and nonsmokers combined.

Figure 3 shows a graph of the incidence rates for females, smokers and nonsmokers, as well as the observed population rates. The population rates are for smokers and nonsmokers combined.

\subsection{Kidney Failure}

Kidney failure is one of the end points covered under CI policies. The U.K. insurance industry's definition of kidney failure is "End-stage renal failure (ESRD) presenting as chronic irreversible failure of both kidneys to function, as a result of which either regular renal dialysis or renal transplant is initiated." See Dinani et al. (2000). The two forms of treatment for ESRD mentioned in the definition are referred to as renal replacement therapy (RRT).

The American Diabetes Association's Clinical Practice Recommendations 2000 state that nephropathy leading to kidney failure is one of the long-term complications of diabetes mellitus. Our model for

Table 2

\section{Coefficients for Fitting Lung Cancer Incidence}

\begin{tabular}{|c|c|c|}
\hline Coefficient & Males & Females \\
\hline$\alpha_{0}$ & -64.09 & -62.014 \\
$\alpha_{1}$ & 20.74 & -1.394 \\
$\alpha_{2}$ & -1.611 & -5.985 \\
$\beta_{0}$ & -191.24 & 31.642 \\
$\beta_{1}$ & 83.155 & -7.729 \\
\hline$\beta_{2}$ & -9.27 & \\
\hline
\end{tabular}


Figure 2

\section{Modeled Incidence Rates (Transition Intensities) of Lung Cancer in Males}

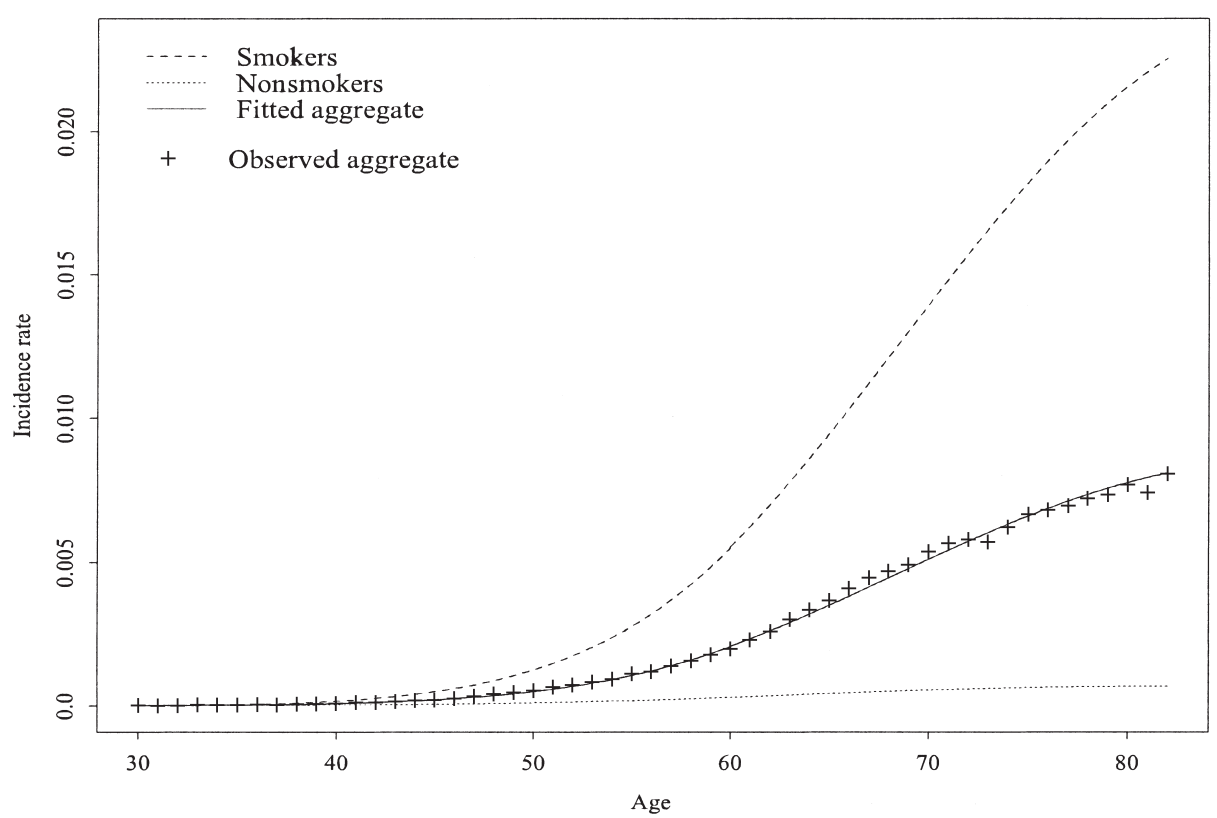

CHD, stroke, and CI explicitly models the influence of diabetes on insurance costs, and therefore we need to consider the difference between incidence rates of kidney failure for diabetics and for nondiabetics. In simple terms, the intensity of entering the "Other CI" state should be higher for diabetics, to allow for the extra risk of kidney failure, than for nondiabetics. Stephens et al. (1990) report that the

Figure 3

\section{Modeled Incidence Rates (Transition Intensities) of Lung Cancer in Females}

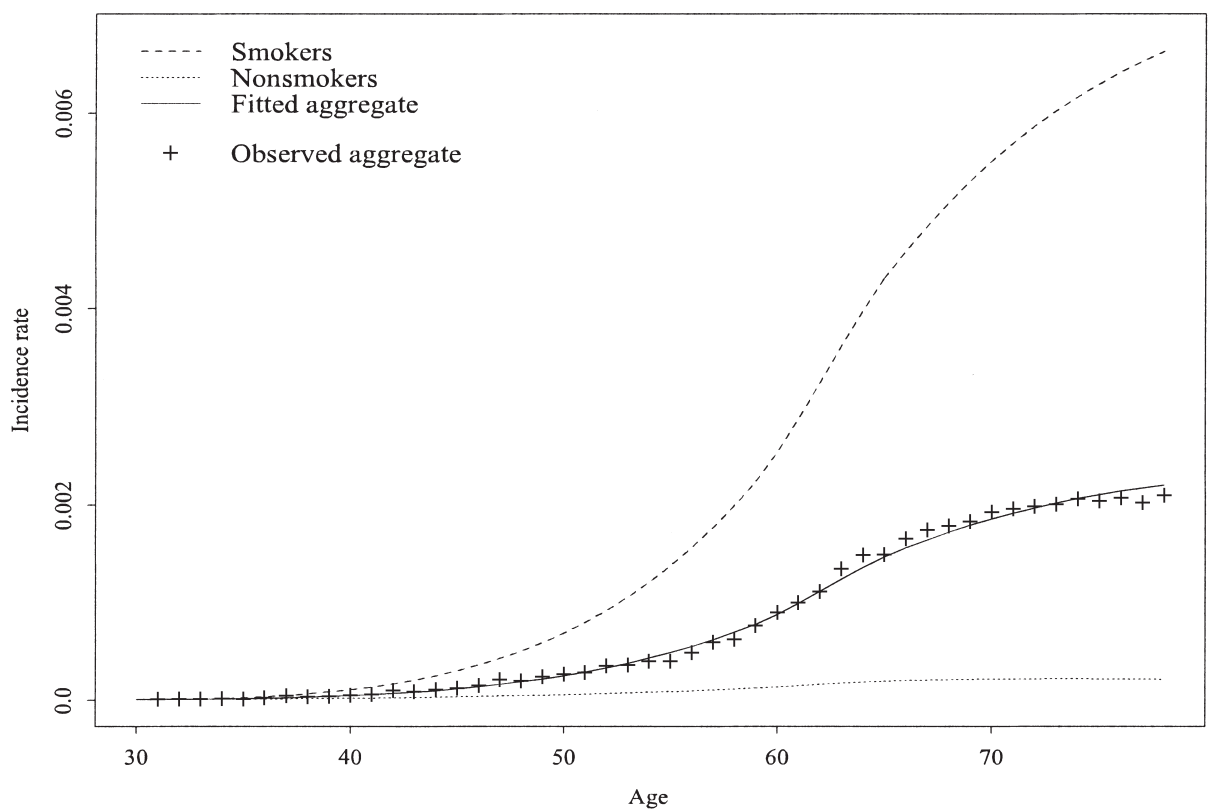


incidence of kidney failure is higher for Type 1 (insulin-dependent) diabetics than for Type 2 (noninsulin-dependent) diabetics.

Data from Harris and Robbins (1994) indicate that the proportion of diabetes cases diagnosed after age 30 which are of Type 1 is about 0.085 , with the remainder, 0.915 , being of Type 2 . These data relate to males and females in the years 1976-80. In our model we will assume that for a life aged $x$ the intensity of developing Type 1 (resp. Type 2) diabetes is $0.085 \lambda_{x}^{\text {diab }}\left(\right.$ resp. $0.915 \lambda_{\infty}^{\text {diab }}$ ), where $\lambda_{\infty}^{\text {diab }}$ is as described in Part I, Section 8.1. In terms of Figure 1, this means that each box labeled "Diab" actually represents two boxes, one for Type 1 diabetes and one for Type 2 diabetes. We assume that no transitions take place between these states, so that, for example, once a life has developed Type 1 diabetes it is not possible subsequently to develop Type 2 diabetes.

For each of Type 1 and Type 2 diabetes, we require estimates of the intensity of developing ESRD, higher in the former case. We estimated the intensity of ESRD by the following function:

$$
\lambda_{x}^{\text {esrd }}=\exp \left(\alpha_{0}+\alpha_{1} x+\alpha_{2} x^{2}+\alpha_{3} x^{3}\right)
$$

where the coefficients for the six combinations of sex and diabetic status are given in Table 3. Graphs of these intensities are shown as Figure 4.

The data sources used to obtain these estimated incidence rates of ESRD were the following:

a. U.S. Renal Data System Annual Data Reports for 1997-2000 (USRDS 1997, 1998, 1999 and 2000). These give, for 1994-97, and for selected states in the United States, the number of new cases of patients requiring RRT subdivided by the primary disease causing the ESRD. (Diabetes is the largest single cause of ESRD.) They also give the corresponding population figures for these states. Finally, they give data relating to the proportion of ESRD cases among diabetics that are attributable to Type 1 diabetics. All these data are for males and females combined. There is evidence in these reports that the number of cases of Type 2 diabetes is increasing so that the proportion of Type 1 cases is falling at all ages.

b. Harris et al. (1998). This gives the prevalence of diabetes in a sample of lives representative of the U.S. population, based on a survey carried out between 1988 and 1994. The prevalence is based on diagnosed diabetes and undiagnosed diabetes. Undiagnosed diabetes is defined as blood sugar level exceeding $126 \mathrm{mg} / \mathrm{dL}$ in lives without a previous diagnosis of diabetes. The definition is comparable with the definition of diabetes that we use in our modeling.

Full details of the estimation procedure used can be found in Wekwete (2002).

Table 3

Coefficients for Fitting Kidney Failure Incidence

\begin{tabular}{|c|c|c|c|}
\hline Coefficient & Nondiabetics & $\begin{array}{c}\text { Type } 1 \\
\text { Diabetics }\end{array}$ & $\begin{array}{c}\text { Type } 2 \\
\text { Diabetics }\end{array}$ \\
\hline \multicolumn{4}{|c|}{ Males } \\
\hline $\begin{array}{l}\alpha_{0} \\
\alpha_{1} \\
\alpha_{2} \\
\alpha_{3}\end{array}$ & $\begin{array}{c}-11.5513 \\
6.509 \times 10^{-2} \\
- \\
-\end{array}$ & $\begin{aligned} & 4.3868 \\
&-5.689 \times 10^{-1} \\
& 1.103 \times 10^{-2} \\
&-6.952 \times 10^{-5}\end{aligned}$ & $\begin{array}{r}- \\
-4.194 \times 10^{-1} \\
8.330 \times 10^{-3} \\
-5.136 \times 10^{-5}\end{array}$ \\
\hline \multicolumn{4}{|c|}{ Females } \\
\hline $\begin{array}{l}\alpha_{0} \\
\alpha_{1} \\
\alpha_{2} \\
\alpha_{3}\end{array}$ & $\begin{array}{c}-12.1810 \\
6.489 \times 10^{-2} \\
- \\
-\end{array}$ & $\begin{array}{c}3.6856 \\
-5.675 \times 10^{-1} \\
1.087 \times 10^{-2} \\
-6.491 \times 10^{-5}\end{array}$ & $\begin{array}{c}-8.9406 \\
4.141 \times 10^{-2} \\
- \\
-\end{array}$ \\
\hline
\end{tabular}


Figure 4

Fitted Incidence Rates of ESRD for Males and Females
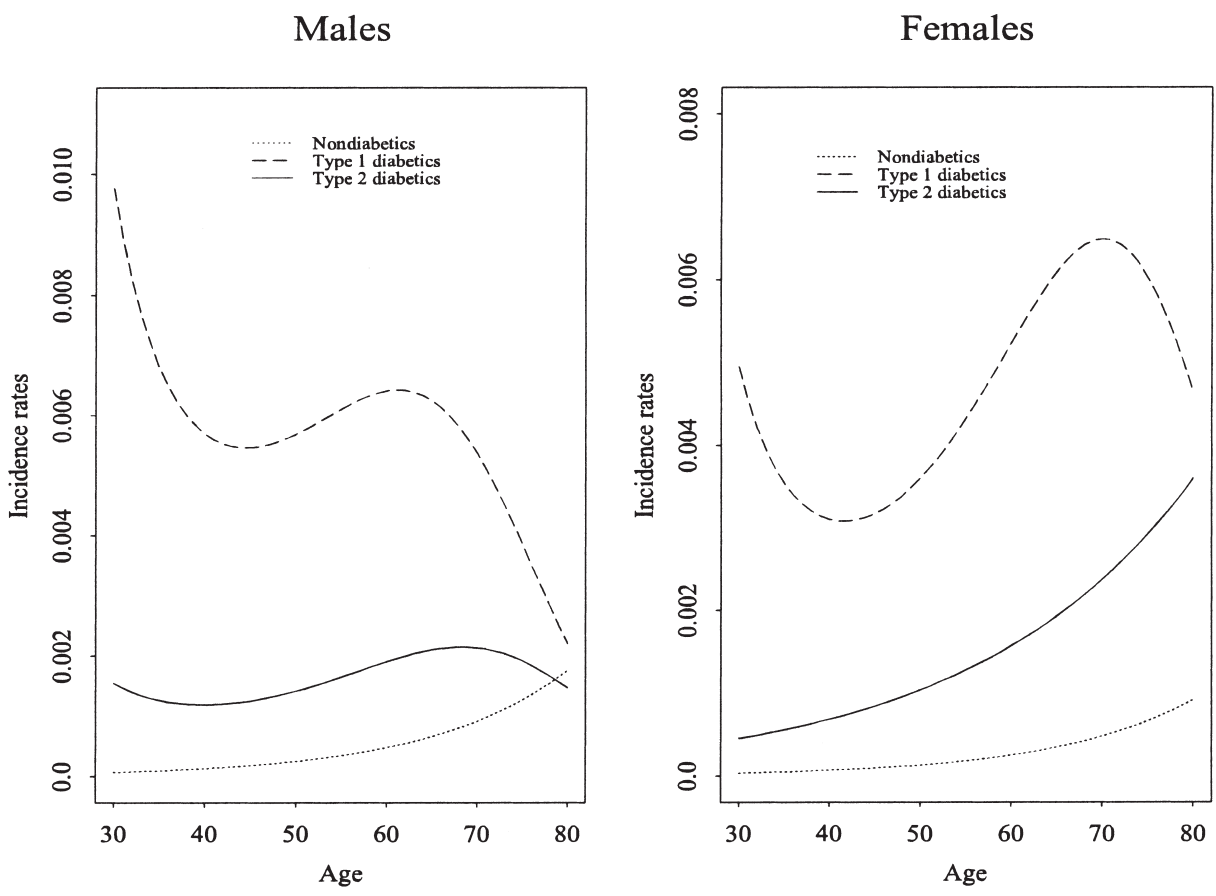

\subsection{Minor CI Claim Causes}

We assume that the (age-dependent) intensities of the causes of CI claims other than CHD, stroke, cancers, and ESRD for males are 20\% of the total of the intensities for CHD, stroke, and cancers. The corresponding figure for females is $15 \%$. These figures are broadly in line with the data on CI claims by cause in Dinani et al. (2000, Appendix 4c). Hence, for example, the intensity $\lambda_{x}^{\text {Other }}$ in Figure 1 for male, nonsmokers is given by

$$
\lambda_{x}^{\text {Other }}=1.2 \times\left(\lambda_{x}^{\text {othercan_males }}+\lambda_{x}^{\text {lung_males_non-sm }}\right)+0.2 \times\left(\lambda_{x}^{\text {CHD }}+\lambda_{x}^{\text {Stroke }}\right)+\lambda_{x}^{\text {esrd }} .
$$

\section{The Force of Mortality}

In our model illustrated as Figure 1, "Dead" means death before the diagnosis of a critical illness. The mortality intensity is taken as that of English Life Table No. 15, Males or Females as appropriate, adjusted for deaths due to diseases whose diagnosis would trigger a CI claim. The adjustment depends on age and sex, but not on any of the other risk factors. Our model for the force of mortality for females, for example, is

$$
\lambda_{x}^{D}=\left(1-\phi_{x}^{f}\right) \lambda_{x}^{E L T 15 F},
$$

where $\lambda_{x}^{E L T 15 F}$ is the force of mortality for females at age $x$ taken from ELT15 and $\phi_{x}^{f}$ is a smoothed estimate of the proportion of deaths at age $x$ among females that are due to cancer, heart attack, stroke, kidney failure, multiple sclerosis, Alzheimer's disease, Parkinson's disease, and benignn brain tumor. Details of the proportions of deaths at each age and for each sex due to these causes in the years 1990-92 were taken from OPCS $(1991,1993 a, 1993 b)$. The functions $\phi_{x}$ were fitted using unweighted least squares and are as follows: 


$$
\begin{aligned}
& \phi_{x}^{f}= \begin{cases}-2.6129 \times 10^{-2}+1.0464 \times 10^{-1} \times x-1.1814 \times 10^{-2} \times x^{2}+4.6714 \times 10^{-4} \times x^{3} & \\
-5.7901 \times 10^{-6} \times x^{4} & \text { for } x \leq 30 \\
-1.3451+8.9722 \times 10^{-2} \times x-1.1998 \times 10^{-3} \times x^{2}+4.8678 \times 10^{-6} \times x^{3} & \text { for } x>35 .\end{cases} \\
& \phi_{x}^{m}= \begin{cases}1.8541 \times 10^{-2}+6.5572 \times 10^{-2} \times x-6.6711 \times 10^{-3} \times x^{2} & : x \leq 30 \\
+2.2397 \times 10^{-4} \times x^{3}-2.2836 \times 10^{-6} \times x^{4} & : x \geq 44 \\
-2.0969+1.0683 \times 10^{-1} \times x-1.2252 \times 10^{-3} \times x^{2}+4.0118 \times 10^{-6} \times x^{3} & : x \geq 0\end{cases}
\end{aligned}
$$

with linear blending between ages 30 and 35 for females and ages 30 and 44 for males.

\section{Premium Rates for Critical Illness Insurance}

\subsection{Preliminaries}

In this section and in Section 6 we will use the model shown in Figure 1, together with the parameterizations summarized or described in Sections 8, 9, and 10 of Part I and in Sections 3 and 4 here, to calculate premiums and related costs associated with CI policies. (Note that we do not incorporate the adjustments to the intensities described in Section 14 of Part 1 . We will comment on the effect of these adjustments in Section 5.5.) Typically we will consider a new policyholder who belongs to one of our 12 subpopulations and who starts in one of the 36 transient states of the model. (Recall that each of the "Diabetes" boxes in Figure 1 represents two states.) This person pays premiums continuously at constant rate until the earliest of death, diagnosis of a critical illness, or end of the policy term. A lump sum is payable on the diagnosis of a critical illness, but not on death or on survival to the end of the policy term.

Norberg (1995) has shown how to solve the simultaneous differential equations satisfied by the moments of the present values of the premium income and of the benefit payments for our model, and hence how to calculate the net premium rate. When calculating present values, we will always use a force of interest of $5 \%$ per annum.

A feature of CI policies in the United Kingdom, and in some other countries, is the 28-day survival rule. Under this rule, no benefit is payable on diagnosis of a critical illness unless the life survives for at least 28 days after diagnosis. This is not a significant condition for critical illnesses such as cancers or ESRD, where death does not usually occur soon after diagnosis, but it is a significant factor for CHD and stroke. Using data, based on insured lives and split by age but not by sex, supplied to us by SwissRe, we derived the following probabilities for 28-day survival following a CHD event or a stroke for a person aged $x$ :

$$
\begin{aligned}
p_{x}^{C H D} & =0.8983095-0.00235911 x-0.00001359781 x^{2}, \\
p_{x}^{\text {stroke }} & =0.8718412+0.001566578 x-0.00003711161 x^{2} .
\end{aligned}
$$

We assume these probabilities apply to both sexes. These survival factors are a little higher than those reported by Morrison et al. (1997) for CHD based on a community study in Glasgow, and by Vemmos et al. (1999) for stroke based on a study carried out in southern Greece. This is discussed in detail in Wekwete (2002, Section 3.1.3).

We can incorporate this 28-day survival rule into our financial calculations by assuming the GHD intensity in Figure $1, \lambda_{x}^{C H D}$, is the intensity derived in Part 1, Section 9, multiplied by $p_{x}^{C H D}$. The remaining part of the CHD intensity, $\lambda_{x}^{C H D} \times\left(1-p_{x}^{C H D}\right)$, is added to the force of mortality. Note that no benefit is payable on death. We adjust the intensity of stroke in a similar fashion. These adjustments have been made to our model for all the calculations in the remaining sections of this paper.

\subsection{The Base Case}

Table 4 shows premium rates, payable continuously and expressed in dollars per annum, for six combinations of age and term for CI cover of $\$ 100,000$ for a nonsmoking male with normal BMI who has 
Table 4

\section{Premium Rates Per Annum for $\mathrm{Cl}$ Cover of $\$ \mathbf{1 0 0 , 0 0 0}$ for Males, Nonsmokers,} Normal BMI, No Additional Risk Factors

\begin{tabular}{|c|c|c|c|c|c|c|}
\hline & \multicolumn{3}{|c|}{ Age 35 at Entry } & \multicolumn{2}{|c|}{ Age 45 at Entry } & \multirow{2}{*}{$\begin{array}{c}\text { Age } 55 \\
\text { at Entry } \\
\text { Term } \\
10 \mathrm{Yr}\end{array}$} \\
\hline & $\begin{array}{l}\text { Term } \\
10 \mathrm{Yr}\end{array}$ & $\begin{array}{l}\text { Term } \\
20 \mathrm{Yr}\end{array}$ & $\begin{array}{l}\text { Term } \\
30 \mathrm{Yr}\end{array}$ & $\begin{array}{l}\text { Term } \\
10 \mathrm{Yr}\end{array}$ & $\begin{array}{l}\text { Term } \\
20 \mathrm{Yr}\end{array}$ & \\
\hline Level net premium & 202 & 322 & 487 & 489 & 745 & 1,124 \\
\hline $\begin{array}{l}\text { Standard deviation of } \\
\text { present value of loss }\end{array}$ & 11,000 & 15,100 & 17,349 & 16,971 & 22,472 & 25,239 \\
\hline $\begin{array}{l}\text { Skewness of present } \\
\text { value of loss }\end{array}$ & 6.96 & 3.87 & 2.59 & 4.34 & 2.35 & 2.66 \\
\hline $\begin{array}{l}\text { Mean of simulated } \\
\text { premiums }\end{array}$ & 204 & 322 & 486 & 487 & 741 & 1,118 \\
\hline $\begin{array}{l}\text { Standard deviation of } \\
\text { simulated premiums }\end{array}$ & 28 & 40 & 57 & 59 & 86 & 129 \\
\hline
\end{tabular}

no additional risk factors, that is, who does not have any of hypertension, hypercholesterolemia, and diabetes and so starts in State 0 in Figure 1. Throughout this section we will regard this life as a "base case" in the sense that premiums for lives with additional risk factors or from other subpopulations will be expressed as percentage additions to or subtractions from the corresponding premium in Table 4 .

The premiums in Table 4 have been calculated using the equivalence principle, so that the expected value of the present value of the loss on the policy is zero. Also shown in this table are the standard deviation and the skewness of the present value of the loss. It can be seen that in each case the skewness is strongly positive, indicating that the distribution of the present value of the loss has a longer right-hand than left-hand tail. This is to be expected since it is possible for the insurer to make a modest negative loss, that is, profit, from a policy with high probability, that is, if no claim is ever made; but it is also possible to make a substantial loss with small probability, that is, if a claim is made.

The premiums in Table 4 are functions of the parameters used to calculate the transition intensities in our model. There are 49 such parameters for both males and females. These parameters are least squares estimates, and we can regard them as the expected values of random variables whose variances and covariances can be obtained from the fitting procedure. Assuming that these random variables have a multivariate normal joint distribution, we can sample from this parameter set, and, for each sampled set of parameters, we can calculate the net premium. We did this 10,000 times for each of the six combinations of age and term shown in Table 4. The sample means and standard deviations of these simulated values are shown in the last two lines of the table. It should be noted that the sample means in each case are close to the net premiums calculated using the mean values of the parameters. This is because the premium, as a function of the 49 parameters, was found to have a reasonably symmetric distribution. The standard deviation of each premium allows us to calculate approximate confidence intervals for the premium by assuming these have a normal distribution. For example, an approximate $95 \%$ confidence interval for the premium for a 35 -year old effecting a 10 -year policy is $(146,258)$, where the limits are calculated as $202 \pm 2 \times 28$. The width of these confidence intervals reflects the amount of data used to estimate the parameters for the transition intensities; the more data we have, the smaller will be the variances of the estimates of these parameters and the tighter will be the confidence intervals.

\subsection{Additional Risk Factors}

Table 5 shows the percentage additions to the "base premium" shown in Table 4 for each of the six combinations of age and term for a life starting in each of the other 35 transient states, that is, with some combination of additional risk factors relating to hypertension, hypercholesterolemia, and diabetes. We are still considering a life from the same subpopulation as in Section 5.2, that is, a nonsmoking male with normal BMI. 
Table 5

Premium Ratings for Males, Nonsmokers, Normal BMI

\begin{tabular}{|c|c|c|c|c|c|c|}
\hline \multirow[b]{2}{*}{ Risk Factors } & \multicolumn{6}{|c|}{ Age at Entry (Term in Years) } \\
\hline & $35(10)$ & $35(20)$ & $35(30)$ & $45(10)$ & $45(20)$ & $55(10)$ \\
\hline $\mathrm{H}^{\prime}$ chol Category 1 & $+3 \%$ & $+5 \%$ & $+5 \%$ & $+3 \%$ & $+4 \%$ & $+2 \%$ \\
\hline Type 1 diabetes & +298 & +185 & +123 & +123 & +83 & +62 \\
\hline Type 2 diabetes & +67 & +46 & +33 & +36 & +27 & +23 \\
\hline Hypertension Category 1 & +6 & +10 & +11 & +6 & +9 & +5 \\
\hline $\mathrm{H}^{\prime}$ chol Category 2 & +25 & +23 & +19 & +23 & +20 & +18 \\
\hline $\mathrm{H}^{\prime}$ chol Category 1 and Type 1 diabetes & +302 & +191 & +129 & +126 & +88 & +64 \\
\hline $\mathrm{H}^{\prime}$ chol Category 1 and Type 2 diabetes & +71 & +52 & +39 & +40 & +31 & +25 \\
\hline $\mathrm{H}^{\prime}$ chol Category 1 and Hypertension Category 1 & +9 & +16 & +18 & +9 & +14 & +7 \\
\hline Hypertension Category 1 and Type 1 diabetes & +305 & +197 & +136 & +130 & +94 & +68 \\
\hline Hypertension Category 1 and Type 2 diabetes & +74 & +58 & +47 & +44 & +38 & +29 \\
\hline Hypertension Category 2 & +34 & +36 & +32 & +32 & +31 & +26 \\
\hline $\mathrm{H}^{\prime}$ chol Category 2 and Type 1 diabetes & +330 & +214 & +146 & +153 & +108 & +85 \\
\hline $\mathrm{H}^{\prime}$ chol Category 2 and Type 2 diabetes & +99 & +75 & +57 & +66 & +51 & +46 \\
\hline $\mathrm{H}^{\prime}$ chol Category 2 and Hypertension Category 1 & +34 & +38 & +35 & +33 & +33 & +26 \\
\hline Hypertension Category $1, \mathrm{H}^{\prime}$ chol Category 1 , Type 1 diabetes & +309 & +205 & +143 & +135 & +100 & +71 \\
\hline Hypertension Category $1, \mathrm{H}^{\prime}$ chol Category 1 , Type 2 diabetes & +78 & +66 & +54 & +48 & +44 & +32 \\
\hline Hypertension Category 2 and $\mathrm{H}^{\prime}$ chol Category 1 & +40 & +45 & +41 & +37 & +37 & +29 \\
\hline Hypertension Category 2 and Type 1 diabetes & +342 & +231 & +162 & +164 & +122 & +95 \\
\hline Hypertension Category 2 and Type 2 diabetes & +111 & +92 & +74 & +78 & +66 & +56 \\
\hline Hypertension Category 3 & +81 & +73 & +60 & +76 & +64 & +64 \\
\hline $\mathrm{H}^{\prime}$ chol Category 2, Hypertension Category 1, Type 1 diabetes & +342 & +232 & +165 & +165 & +124 & +95 \\
\hline $\mathrm{H}^{\prime}$ chol Category 2, Hypertension Category 1, Type 2 diabetes & +111 & +94 & +76 & +78 & +68 & +56 \\
\hline $\mathrm{H}^{\prime}$ chol Category 2 and Hypertension Category 2 & +81 & +78 & +66 & +75 & +66 & +59 \\
\hline Hypertension Category 2, $\mathrm{H}^{\prime}$ chol Category 1 , Type 1 diabetes & +349 & +241 & +172 & +171 & +130 & +99 \\
\hline Hypertension Category 2, $\mathrm{H}^{\prime}$ chol Category 1, Type 2 diabetes & +118 & +103 & +84 & +84 & +74 & +60 \\
\hline Hypertension Category 3 and $\mathrm{H}^{\prime}$ chol Category 1 & +89 & +84 & +71 & +83 & +72 & +69 \\
\hline Hypertension Category 3 and Type 1 diabetes & +407 & +281 & +199 & +224 & +166 & +148 \\
\hline Hypertension Category 3 and Type 2 diabetes & +176 & +142 & +110 & +138 & +110 & +109 \\
\hline Hypertension Category 2, $\mathrm{H}^{\prime}$ chol Category 2, Type 1 diabetes & +402 & +284 & +203 & +219 & +165 & +137 \\
\hline Hypertension Category 2, $\mathrm{H}^{\prime}$ chol Category 2, Type 2 diabetes & +172 & +146 & +115 & +133 & +110 & +98 \\
\hline Hypertension Category 3 and $\mathrm{H}^{\prime}$ chol Category 2 & +147 & +129 & +102 & +135 & +110 & +110 \\
\hline Hypertension Category 3, $\mathrm{H}^{\prime}$ chol Category 1 , Type 1 diabetes & +416 & +294 & +211 & +233 & +176 & +153 \\
\hline Hypertension Category 3, $\mathrm{H}^{\prime}$ chol Category 1 , Type 2 diabetes & +185 & +156 & +123 & +147 & +120 & +114 \\
\hline Hypertension Category 3, H'chol Category 2, Type 1 diabetes & +491 & +351 & +250 & +301 & +223 & +206 \\
\hline Hypertension Category 3, $\mathrm{H}^{\prime}$ chol Category 2, Type 2 diabetes & +260 & +213 & +163 & +214 & +168 & +167 \\
\hline
\end{tabular}

Features of this table are the following:

a. The most significant single risk factor is Type 1 diabetes, followed by hypertension, Category 3 .

b. Type 1 diabetes is far more significant than Type 2 diabetes.

c. The effect of combining risk factors is closer to being additive than to being multiplicative. For example, consider a 35-year old who effects a 10-year policy. The individual ratings for "Hypertension, Category 2," "Hypercholesterolemia, Category 1," and "Type 2 Diabetes" are 34\%, 3\%, and 67\%, respectively. Combined, these risk factors give a rating of $118 \%$, which is closer to $104 \%(=34+3+$ $67)$ than to $230 \%(=1.34 \times 1.03 \times 1.67)$.

d. There are many apparent synergies in the table. For example, the rating for the combination of risk factors above, $118 \%$, is greater than the sum of the individual ratings, $104 \%$. Such apparent synergies may not be statistically significant.

We compared the ratings in Table 5 with those in the underwriting manual of a major reinsurer. There was broad agreement between the two sets of ratings. The only risk factor for which there was a significant discrepancy was Type 2 diabetes, where the ratings in Table 5 were lower, sometimes considerably lower, than those in the underwriting manual. Care should be exercised in making these comparisons; the ratings in Table 5 are additions to the lowest possible premium (for the given subpopulation, age and term), whereas an underwriting manual will give ratings as additions to the 
premium for a "standard" life. For a given subpopulation, age, and term, the class of "standard" lives may include lives with one or more of the additional risk factors in our model.

\subsection{Other Subpopulations}

Table 6 shows the percentage addition to, or subtraction from, the corresponding premium in Table 4, for a life, without any additional risk factors, from each of the other 11 subpopulations.

Features to note from Table 6 are the following:

a. BMI has relatively little effect on premium rates.

b. The synergy between risk factors observed in Table 5 also holds across subpopulations. For example, for males with age at entry of 35 years and policy term of 10 years, the rating for hypertension Category 3 is $+81 \%$ (Table 5 ), the rating for smokers is $+24 \%$ (Table 6 ), but the rating for smokers with hypertension, Category 3 , is $+139 \%$ (not shown).

\subsection{Adjustments to the Intensities}

In Section 15 of Part I, we proposed some adjustments to the intensities for hypercholesterolemia (both sexes) and hypertension (females only) to bring the predicted prevalence rates for these conditions more into line with recent U.K. experience. For both sets of intensities, the adjustments were reductions.

Incorporating these adjustments reduces the premium rates shown in Table 4, but only marginally; the largest reduction is from $\$ 487$ to $\$ 480$ for a 35 -year-old who effects a 30-year policy. The ratings for additional risk factors for nonsmoking males with normal BMI calculated using the adjusted intensities are all very close to those shown in Table 5; the largest differences among the 210 values are one of $+5 \%$ and one of $-5 \%$.

The adjustments are more extensive for females. Nevertheless, the same pattern was observed: a very modest reduction in premium rates for all six combinations of age and term and very minor changes in the premium ratings for all 36 combinations of risk factors for age 35 , term 10 years.

\section{Genetics}

Our aim in this paper and in Part I has been to develop a CI model that could be used to assess the impact on insurance underwriting of genetic information relevant to CHD and/or stroke. As explained in Section 4 of Part I, these conditions are thought to be multifactorial so that there is unlikely to be any

Table 6

Premium Ratings for Other Subpopulations

\begin{tabular}{|c|c|c|c|c|c|c|}
\hline \multirow[b]{2}{*}{ Subpopulation } & \multicolumn{3}{|c|}{ Age 35 at Entry } & \multicolumn{2}{|c|}{ Age 45 at Entry } & \multirow{2}{*}{$\begin{array}{c}\text { Age } 55 \text { at } \\
\text { Entry } \\
\text { Term } \\
10 \mathrm{Yr}\end{array}$} \\
\hline & $\begin{array}{l}\text { Term } \\
10 \mathrm{Yr}\end{array}$ & $\begin{array}{l}\text { Term } \\
20 \mathrm{Yr}\end{array}$ & $\begin{array}{l}\text { Term } \\
30 \mathrm{Yr}\end{array}$ & $\begin{array}{l}\text { Term } \\
10 \mathrm{Yr}\end{array}$ & $\begin{array}{l}\text { Term } \\
20 \mathrm{Yr}\end{array}$ & \\
\hline $\begin{array}{l}\text { Males: } \\
\text { Smokers } \\
\text { Overweight } \\
\text { Smokers and overweight } \\
\text { Obese } \\
\text { Smokers and obese } \\
\text { Females } \\
\text { Smokers } \\
\text { Overweight } \\
\text { Smokers and overweight } \\
\text { Obese } \\
\text { Smokers and obese }\end{array}$ & $\begin{array}{l}+24 \% \\
+0 \\
+24 \\
+2 \\
+25 \\
+19 \\
+33 \\
+19 \\
+33 \\
+19 \\
+34\end{array}$ & $\begin{array}{l}+34 \% \\
+1 \\
+35 \\
+2 \\
+37 \\
+10 \\
+30 \\
+10 \\
+31 \\
+12 \\
+32\end{array}$ & $\begin{array}{l}+43 \% \\
+1 \\
+44 \\
+3 \\
+46 \\
-4 \\
+22 \\
-3 \\
+23 \\
-1 \\
+25\end{array}$ & $\begin{array}{l}+43 \% \\
+0 \\
+43 \\
+1 \\
+44 \\
+9 \\
+33 \\
+9 \\
+33 \\
+10 \\
+34\end{array}$ & $\begin{array}{l}+52 \% \\
+1 \\
+53 \\
+2 \\
+55 \\
-8 \\
+21 \\
-7 \\
+22 \\
-6 \\
+23\end{array}$ & $\begin{array}{l}+65 \% \\
+0 \\
+66 \\
+1 \\
+67 \\
-19 \\
+13 \\
-19 \\
+13 \\
-18 \\
+14\end{array}$ \\
\hline
\end{tabular}


single gene for which a mutation gives a significantly higher risk of disease, but there is some evidence of genetic links related to the risk factors for these conditions, notably diabetes, hypercholesterolemia, and hypertension. In this section we make some assumptions concerning genetic links either to the risk factors or directly to CHD and stroke. By calculating the increase in premiums for these scenarios we can increase our understanding of the likely financial effects resulting from direct or indirect genetic links to CHD and stroke that have yet to be determined. Further numerical examples can be found in Wekwete (2002).

For ease of presentation, all our numerical calculations in this section relate to a nonsmoking male aged 35 with normal BMI who effects a CI policy with a term of 10 years. We will use our model with the unadjusted intensities as a baseline so that the premium ratings in this section can be compared with those in Table 5, and all ratings are relative to the premium of $\$ 202$ shown in Table 4 .

Table 7 shows the premium ratings for each of the 36 combinations of risk factors assuming that the set of intensities for each of the risk factors in turn is multiplied by 5 . For example, the figures in the column headed "Chol" are the premium ratings, given a base premium of $\$ 202$, assuming the two intensities $\lambda_{x}^{\text {chol01 }}$ and $\lambda_{x}^{\text {chol12 }}$, as parameterized in Section 8 of Part I, are both multiplied by 5 , with the other intensities unchanged. The figures in the column headed "None" are taken directly from Table 5

Table 7

Premium Ratings for Males, Nonsmokers, Normal BMI, Aged 35 at Entry with Policy Term 10 Years, under Hypothetical Assumptions of Genetic Influence Increasing Incidence Risk Factors Five Times

\begin{tabular}{|c|c|c|c|c|c|}
\hline \multirow[b]{2}{*}{ Risk Factors } & \multicolumn{5}{|c|}{ Premium Rating Factors with $5 \times$ the Incidence Rate of } \\
\hline & None & $\mathrm{H}^{\prime}$ chol & Hypertension & $\begin{array}{c}\text { Type } 1 \\
\text { Diabetes }\end{array}$ & $\begin{array}{c}\text { Type } 2 \\
\text { Diabetes }\end{array}$ \\
\hline 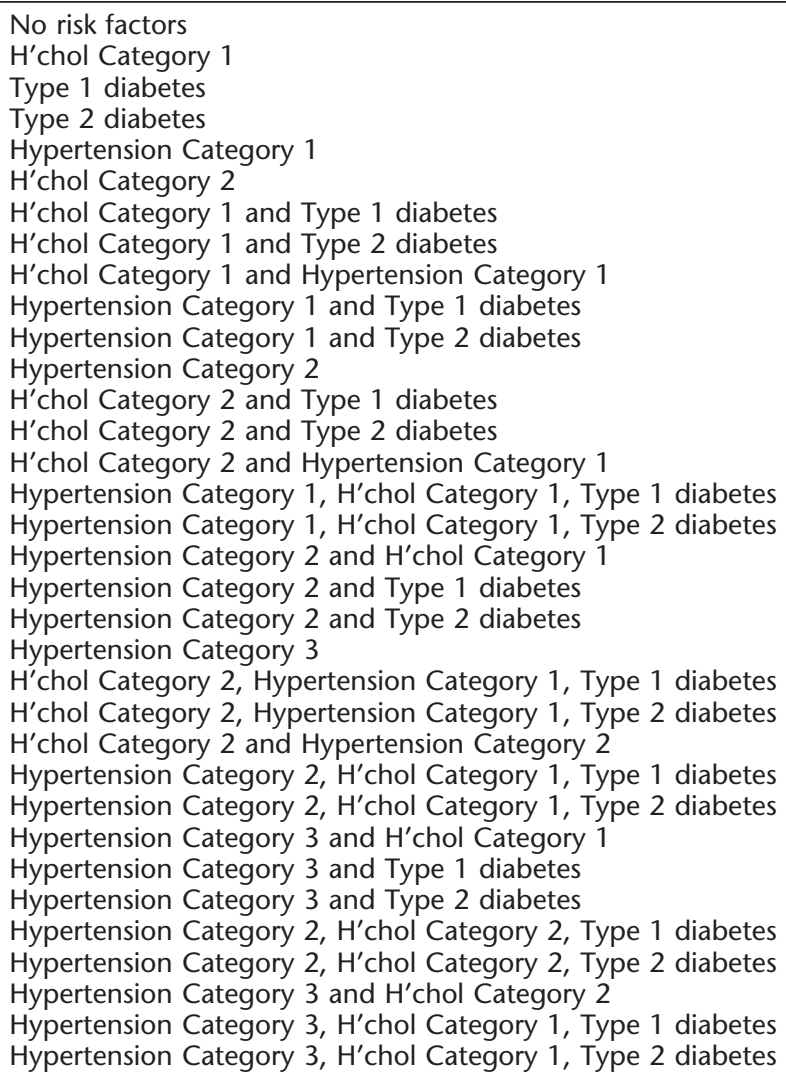 & 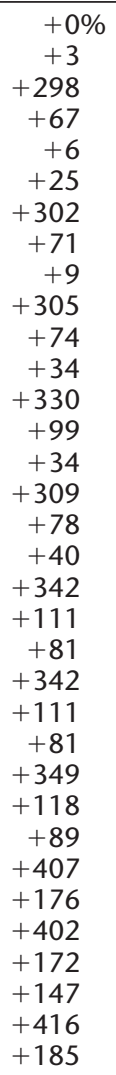 & $\begin{array}{c}+5 \% \\
+12 \\
+304 \\
+73 \\
+12 \\
\\
+313 \\
+82 \\
+19 \\
+313 \\
+82 \\
+44\end{array}$ & $\begin{array}{l}+13 \% \\
+17 \\
+314 \\
+84 \\
+29 \\
+45 \\
+320 \\
+89 \\
+34 \\
+336 \\
+105 \\
+53 \\
+356 \\
+125 \\
+70 \\
+342 \\
+112 \\
+60 \\
+368 \\
+137 \\
+ \\
+38 \\
+158 \\
+108 \\
+376 \\
+146\end{array}$ & $\begin{array}{l}+2 \% \\
+5 \\
+8 \\
+28 \\
+11 \\
+37 \\
+37 \\
+42 \\
+84 \\
+84 \\
+91\end{array}$ & $\begin{array}{l}+47 \\
+90\end{array}$ \\
\hline
\end{tabular}


and are included for comparison. Note that where the life starts with one of the risk factors at the highest level, for example, "Hypertension Category 3," there is no (additional) effect from increasing the intensities of this risk factor, and so no rating is shown.

The additional ratings shown in Table 7 are generally modest. Table 8 shows the effect of multiplying the intensities of the risk factors by 50 . The effects are more significant. However, increasing risks by a factor of 50 is extreme; this is in line with the maximum extra risk of breast cancer conveyed by a mutation in the BRCA1 gene. See Macdonald, Waters, and Wekwete (2003a, 2003b).

Tables 7 and 8 show the effect on CI premium rates of an increase in the risk of one of the risk factors for CHD and stroke. In these cases the influence of the hypothetical genetic information on CHD and stroke is indirect, that is, through one of the risk factors. In Table 9 we show the effect of increasing the intensity of a CHD event or of a stroke in each case by a factor of 5. In particular, the premium ratings in the columns headed "CHD," resp. "Stroke," have been calculated assuming all the intensities $\lambda_{x}^{C H D}$, resp. $\lambda_{x}^{\text {Stroke }}$, in our model, as parameterized in Section 9, resp. Section 10, of Part I have been multiplied by 5 . In the final four columns of this table, we assume that the multiplier of 5 applies to the intensities of CHD, but only when the life has already developed one of the risk factors beyond the base level. For example, in the column headed "Hypertension" we assume that the intensity of a CHD event is multiplied by 5 for a life who has Hypertension Category 1, 2, or 3.

Table 8

Premium Ratings for Males, Nonsmokers, Normal BMI, Aged 35 at Entry with Policy Term 10 Years, under Hypothetical Assumptions of Genetic Influence Increasing Incidence of Risk Factors 50 Times

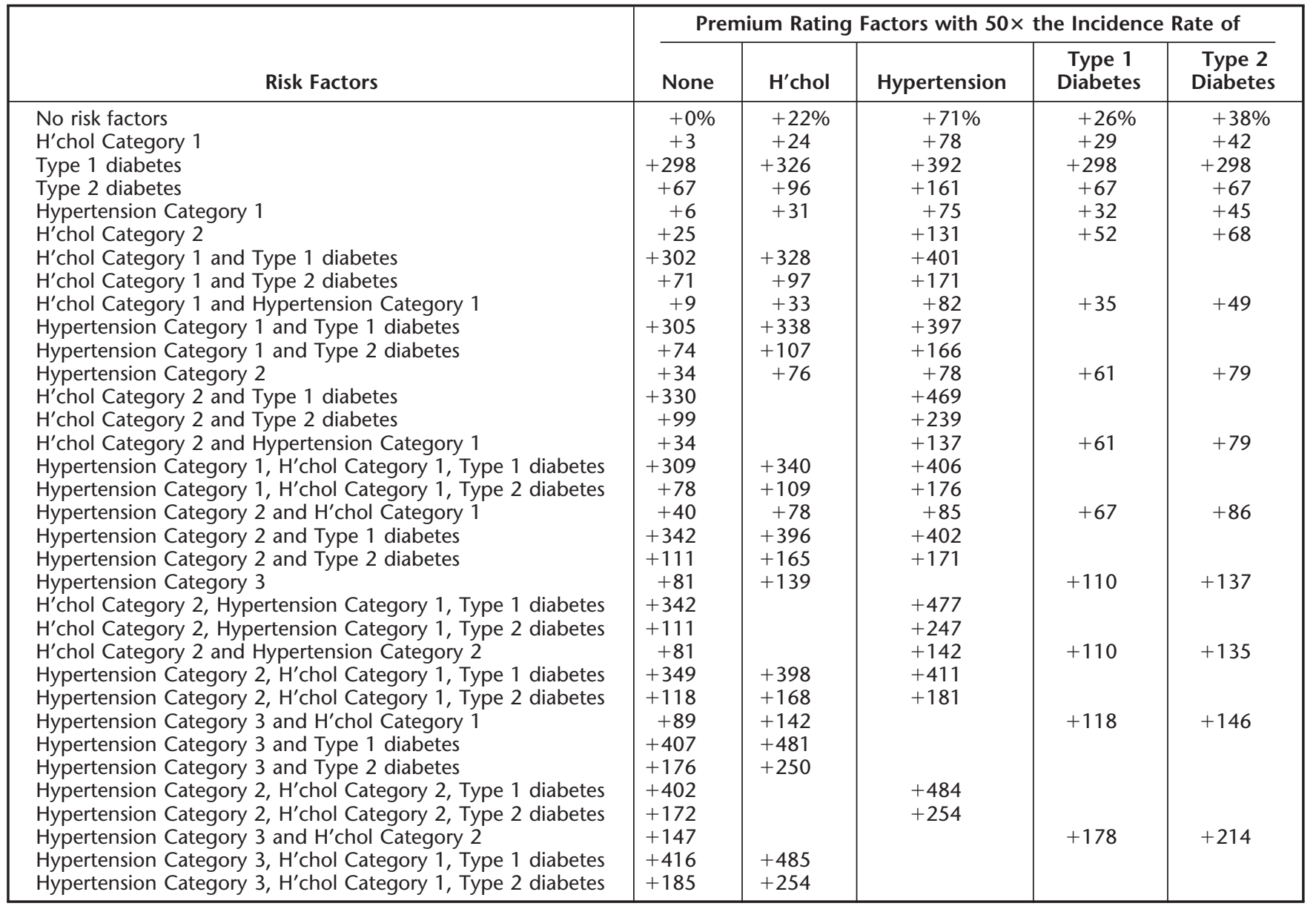


Table 9

\section{Premium Ratings for Males, Nonsmokers, Normal BMI, Aged 35 at Entry with Policy Term 10 Years, under Hypothetical Assumptions of Genetic Influence Increasing Incidence of CHD and Stroke Five Times}

\begin{tabular}{|c|c|c|c|c|c|c|c|}
\hline \multirow[b]{3}{*}{ Risk Factors } & \multicolumn{7}{|c|}{ Premium Rating Factors with $5 \times$ the Incidence Rate of } \\
\hline & \multirow[b]{2}{*}{ None } & \multirow[b]{2}{*}{ CHD } & \multirow[b]{2}{*}{ Stroke } & \multicolumn{4}{|c|}{ CHD Modified by the Presence of } \\
\hline & & & & $\mathrm{H}^{\prime} \mathrm{chol}$ & Hypertension & $\begin{array}{c}\text { Type } 1 \\
\text { Diabetes }\end{array}$ & $\begin{array}{c}\text { Type } 2 \\
\text { Diabetes }\end{array}$ \\
\hline No risk factors & $+0 \%$ & $+142 \%$ & $+37 \%$ & $+26 \%$ & $+29 \%$ & $+1 \%$ & $+5 \%$ \\
\hline $\mathrm{H}^{\prime}$ chol Category 1 & +3 & +156 & +40 & +157 & +35 & +4 & +8 \\
\hline Type 1 diabetes & +298 & +481 & +351 & +331 & +334 & +481 & \\
\hline Type 2 diabetes & +67 & +250 & +121 & +100 & +104 & & +250 \\
\hline Hypertension Category 1 & +6 & +168 & +44 & +36 & +168 & & +11 \\
\hline $\mathrm{H}^{\prime}$ chol Category 2 & +25 & +267 & +62 & +267 & +74 & +26 & +33 \\
\hline $\mathrm{H}^{\prime}$ chol Category 1 and Type 1 diabetes & +302 & +499 & +355 & +499 & +342 & +499 & \\
\hline $\mathrm{H}^{\prime}$ chol Category 1 and Type 2 diabetes & +71 & +268 & +124 & +268 & +111 & & +268 \\
\hline $\begin{array}{l}\text { H'chol Category } 1 \text { and Hypertension } \\
\text { Category } 1\end{array}$ & +9 & +184 & +47 & +184 & +184 & +10 & +15 \\
\hline Hypertension Category 1 and Type 1 & & & & & & & \\
\hline $\begin{array}{l}\text { diabetes } \\
\text { Hypertension Category } 1 \text { and Type } 2\end{array}$ & +305 & +513 & +361 & +343 & +513 & +513 & \\
\hline diabetes & +74 & +283 & +130 & +113 & +283 & & +283 \\
\hline Hypertension Category 2 & +34 & +298 & +83 & +82 & +298 & +35 & +43 \\
\hline $\mathrm{H}^{\prime}$ chol Category 2 and Type 1 diabetes & +330 & +641 & +384 & +641 & +392 & +641 & \\
\hline $\mathrm{H}^{\prime}$ chol Category 2 and Type 2 diabetes & +99 & +411 & +153 & +411 & +161 & & +411 \\
\hline $\mathrm{H}^{\prime} \mathrm{chol}$ Category 2 and Hypertension & & & & & & & \\
\hline Category 1 & +34 & +309 & +73 & +309 & +309 & +35 & +44 \\
\hline $\begin{array}{l}\text { Hypertension Category } 1, \mathrm{H}^{\prime} \text { chol Category } 1 \text {, } \\
\text { Type } 1 \text { diabetes }\end{array}$ & +309 & +534 & +365 & +534 & +534 & +534 & \\
\hline Hypertension Category 1, $\mathrm{H}^{\prime}$ chol Category 1 , & & & & & & & \\
\hline Type 2 diabetes & +78 & +304 & +134 & +304 & +304 & & +304 \\
\hline $\begin{array}{l}\text { Hypertension Category } 2 \text { and } \mathrm{H}^{\prime} \text { chol } \\
\text { Category } 1\end{array}$ & +40 & +324 & +89 & +324 & +324 & & +49 \\
\hline Hypertension Category 2 and Type 1 & & & TOY & $T 324$ & $T 324$ & & T4 45 \\
\hline diabetes & +342 & +681 & +414 & +403 & +681 & +681 & \\
\hline $\begin{array}{l}\text { Hypertension Category } 2 \text { and Type } 2 \\
\text { diabetes }\end{array}$ & +111 & +451 & +183 & +173 & +451 & & +451 \\
\hline Hypertension Category 3 & +81 & +450 & +213 & +147 & +450 & +82 & +93 \\
\hline $\begin{array}{l}\mathrm{H}^{\prime} \text { chol Category 2, Hypertension Category 1, } \\
\text { Type } 1 \text { diabetes }\end{array}$ & +342 & +694 & +398 & +694 & +694 & +694 & \\
\hline $\mathrm{H}^{\prime}$ chol Category 2, Hypertension Category 1, & & & & & & & \\
\hline Type 2 diabetes & +111 & +465 & +167 & +465 & +465 & & +465 \\
\hline Hypertension Category 2, $\mathrm{H}^{\prime}$ chol Category 1 , & +81 & +528 & +130 & +528 & +528 & +82 & +96 \\
\hline Type 1 diabetes & +349 & +714 & +421 & +714 & +714 & +714 & \\
\hline Hypertension Category 2, $\mathrm{H}^{\prime}$ chol Category 1 , & & & & & & & \\
\hline $\begin{array}{l}\text { Type } 2 \text { diabetes } \\
\text { Hypertension Category } 3 \text { and } \mathrm{H}^{\prime} \text { chol }\end{array}$ & +118 & +485 & +190 & +485 & +485 & & +485 \\
\hline Category 1 & +89 & +485 & +220 & +485 & +485 & +90 & +102 \\
\hline Hypertension Category 3 and Type 1 & +407 & +881 & +598 & +489 & +881 & +881 & \\
\hline $\begin{array}{l}\text { diabetes } \\
\text { Hypertension Category } 3 \text { and Type } 2\end{array}$ & +407 & +881 & +598 & +489 & +881 & +881 & \\
\hline diabetes & +176 & +651 & +368 & +259 & +651 & & +651 \\
\hline Hypertension Category 2, H'chol Category 2, & +402 & +976 & +474 & +976 & +976 & +976 & \\
\hline Hypertension Category 2, H'chol Category 2, & & & & & ТY10 & & \\
\hline $\begin{array}{l}\text { Type } 2 \text { diabetes } \\
\end{array}$ & +172 & +747 & +243 & +747 & +747 & & +747 \\
\hline Hypertension Category 3 and $\mathrm{H}^{\prime} \mathrm{chol}$ & & & & & & & \\
\hline $\begin{array}{l}\text { Category } 2 \\
\text { Hypertension Category 3. H'chol Category } 1\end{array}$ & +147 & +771 & +278 & +771 & +771 & +148 & +166 \\
\hline $\begin{array}{l}\text { Hypertension Category 3, } \mathrm{H}^{\prime} \mathrm{chol} \text { Category I, } \\
\text { Type } 1 \text { diabetes }\end{array}$ & +416 & +925 & +608 & +925 & +925 & +925 & \\
\hline Hypertension Category 3, $\mathrm{H}^{\prime}$ chol Category 1 , & & & & & & & \\
\hline Type 2 diabetes & +185 & +696 & +377 & +696 & +696 & & +696 \\
\hline Hypertension Category 3, H'chol Category 2, & +491 & +1293 & +682 & +1293 & +1293 & +1293 & \\
\hline Hypertension Category 3, $\mathrm{H}^{\prime}$ chol Category 2, & & & & & & 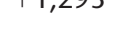 & \\
\hline Type 2 diabetes & +260 & $+1,064$ & +452 & $+1,064$ & $+1,064$ & & $+1,064$ \\
\hline
\end{tabular}


The premium ratings in Table 9 are far in excess of those in Table 7 and even those in Table 8. This indicates, perhaps not surprisingly, that a genetic profile that conveys a higher risk of CHD or of stroke, even if it operates only when one of the risk factors is present, is likely to have a far greater financial effect than a genetic profile that conveys a higher risk of diabetes, hypertension, or hypercholesterolemia, no matter how much greater this risk. However, it should be recalled from Section 4 of Part I that genetic links to CHD and stroke determined to date have all acted through one of our risk factors.

\section{Conclusions}

We have developed a model, shown as Figure 1, for critical illness insurance that incorporates, or includes pathways through, the major risk factors for CHD and stroke. This model is an extension of the model for CHD and stroke developed in Part I of this work; the major extensions are the inclusion of other critical illnesses, notably cancers, as an end point and the detailed modeling of ESRD (kidney failure) for Type 1 and Type 2 diabetics.

Our model has been parameterized, to a large extent, using data from the Framingham Heart Study. This data set is relatively old. In Section 15 of Part I we discussed ways in which our parameter estimates could be adjusted so that the model produced prevalence rates for CHD and stroke more in line with more recent and more relevant experience. Making these adjustments had only a minor effect on premiums and premium ratings produced by our CI model (see Section 5.5).

Our CI model can be used as an underwriting tool. Premium ratings from our model have been compared with those in the underwriting manual of a major reinsurer. There is broad agreement except in the case of Type 2 diabetes, where the ratings from our model were lower than those in the manual (see Section 5.3).

One of our major aims in this work has been to develop a model that can be used to assess the financial effects on insurers, in particular for CI premiums, of genetic information relating to CHD and stroke. At present, genetic links to CHD and stroke, either direct or through risk factors, have not been established to the extent where the financial effects can be determined. However, sample calculations show that genetic links that operate only through risk factors are unlikely to be financially significant (see Section 6).

\section{ACKNOWLEDGments}

This work was funded by Swiss Re Life and Health, to whom we are grateful for financial support and for many discussions with their actuarial, medical, and underwriting staff and consultants. In particular, we wish to thank Douglas Keir, Dr. David Muiry, Dr. Kevin Somerville, and Dr. Hanspeter Würmli.

The authors are grateful to two anonymous referees whose constructive comments helped to improve this paper.

This paper, and Part I, use data supplied by the National Heart, Lung, and Blood Institute, NIH, DHHS. The views expressed in these papers are those of the authors and do not necessarily reflect the views of the National Heart, Lung, and Blood Institute. We also use data supplied by the United States Renal Data Systems (USRDS). The interpretation and reporting of these data are the responsibility of the authors and in no way should be seen as an official policy or interpretation of the U.S. government.

\section{REFERENCES}

American Diabetes Association. 2000. Clinical Practice Recommendations 2000: Report of the Expert Committee on the Diagnosis and Classification of Diabetes Mellitus. Diabetes Care 22, Supplement 1.

Department of Health. 1998. Statistics on Smoking: England, 1976 to 1996. Bulletin 1998/25. Online at www.publications. doh.gov.uk/pub/docs/doh/smoking.pdf.
Dinani, Azim, David Grimshaw, Neil Robjohns, Stephen Somerville, Alasdair Spry, and Jerry Staffurth. 2000. A Critical Reviez: Report of the Critical Illness Healthcare Study Group. Unpublished manuscript presented to the Staple Inn Actuarial Society, London, 14 March 2000.

Doll, Richard 1971. The Age Distribution of Cancer: Implications for Models of Carcinogenesis. Journal of the Royal Statistical Society Series A 134(2): 133-66. 
Harris, Maureen I., Katherine M. Flegal, Catherine G. Cowie, Mark S. Eberhart, David E. Goldstein, Randie R. Little, Hsio-Mei Wiedmeyer, and David D. Byrd-Holt. 1998. Prevalence of Diabetes, Impaired Fasting Glucose and Impaired Glucose Tolerance in U.S. Adults: The Third National Health and Nutrition Examination Survey, 1988-1994. Diabetes Care 21: 518-24.

Harris, Maureen I., and David C. Robbins. 1994. Prevalence of Adult-Onset IDDM in the U.S. Population. Diabetes Care 17: 1337-40.

Macdonald, Angus S., Howard R. Waters, and Chessman T. WeKwete. 2003a. The Genetics of Breast and Ovarian Cancer I: A Model of Family History. Scandinavian Actuarial Journal, 1-27.

- 2003b. The Genetics of Breast and Ovarian Cancer II: A Model of Critical Illness Insurance. Scandinavian Actuarial Journal, 28-50.

Morrison, Garoline, Mark Woodward, Wilma leslie, and Hugh Tustall-Pedoe. 1997. Effect of Socioeconomic Group on Incidence of, Management of, and Survival after Myocardial Infarction and Coronary Death: Analysis of Community Coronary Register. British Medical Journal 314: 541-46.

Norberg, Ragnar. 1995. Differential Equations for Moments of Present Values in Life Insurance. Insurance: Mathematics and Economics 17: 171-80.

ONS. 1998. Cancer 1971-1997. CD ROM supplied by the Office of National Statistics, London.

OPCS. 1991. 1990 Mortality Statistics: Cause. Series DH2 No. 17.

1993a. 1991 Mortality Statistics: Cause. Series DH2 No. 18.

. 1993b. 1992 Mortality Statistics: Cause. Series DH2 No. 19.

Stephens, Gregory W., James A. Gillaspy, Agnes Mejia, and Vic-
TOR E. POLLACK. 1990. Racial Differences in the Incidence of End-Stage Renal Disease in Types I and II Diabetes Mellitus. American Journal of Kidney Disease 15: 562-67.

Swiss Re Underwriting Manual. 1995. Swiss Re Life and Health. London.

USRDS. 1997. United States Renal Data System 1997 Annual Report. Bethesda, Md.: National Institutes of Health, National Institute of Diabetes, Digestive and Kidney Diseases. 1998. United States Renal Data System 1998 Annual Report. Bethesda, Md.: National Institutes of Health, National Institute of Diabetes, Digestive and Kidney Diseases.

1999. United States Renal Data System 1999 Annual Report. Bethesda, Md.: National Institutes of Health, National Institute of Diabetes, Digestive and Kidney Diseases.

- 2000. United States Renal Data System 2000 Annual Report. Bethesda, Md.: National Institutes of Health, National Institute of Diabetes, Digestive and Kidney Diseases.

Vemmos, Konstantinos N., Michiel L. Bots, Panagiotis K. Tsibouris, Vasilios P. Zis, Diederick E. Grobbee, Georgios S. Stranjalis, and Stamatis Stamatelopoulos. 1999. Stroke Incidence and Case Fatality in Southern Greece: The Arcadia Stroke Registry. Stroke 30: 363-70.

Weкwete, Chessman T. 2002. Genetics and Critical Illness Insurance Underwriting: Models for Breast Cancer and Ovarian Cancer and for Coronary Heart Disease and Stroke. Ph.D. thesis, Heriot-Watt University, Edinburgh. Online at www.ma.hw.ac.uk/ams.html.

Discussions on this paper can be submitted until July 1, 2005. The authors reserve the right to reply to any discussion. Please see the Submission Guidelines for Authors on the inside back cover for instructions on the submission of discussions. 